\title{
A Method of 3D Point Cloud Volume Calculation Based on Slice Method
}

\author{
Yu Zhi, Yikun Zhang, Hao Chen, Kaifeng Yang, Hui Xia \\ Faculty of Computer Science and Engineering \\ Xi'an University of Technology \\ Xi'an China \\ ykzhang163@163.com
}

\begin{abstract}
This paper proposes a method for calculating the volume of 3D point cloud based on slice method. Firstly, determine the projection plane. Put the projection plane as a $X O Y$ plane in projection coordinate system, and establish a projection coordinate system, cut the $3 D$ point cloud along $Z$-axis direction projection coordinate system. And all the point cloud data of each cutting block is projected onto the $X O Y$ plane. Then filter the data points using scanning algorithm in this paper, to get area and volume of point cloud. Experiments show that the algorithm can improve the accuracy of volume calculation even its efficiency is with a determined accuracy.
\end{abstract}

Keywords - coordinate transformation; point cloud slice; projection

\section{INTRODUCTION}

The point cloud is a point data set of products obtained by measuring instruments in reverse engineering. Because of the advantages of easy access, simple, flexible and so on, the point cloud has gradually become one of the usual 3D models. The point cloud data usually carry the coordinate information and other topological information, most of the 3D models use point cloud data. This allows the 3D modeling to be applied to more fields. The point cloud volume calculation based on 3D modeling is also applied to all aspects of society.

In the point cloud volume calculation, many scholars have carried out a lot of research: Md Hafizur and Carol Bouchey ${ }^{[1]}$ attempt to estimate the volume which is based on dietary influence system in the mobile terminal, although the paper also use the method, but not the specific details, according to the results, the calculation accuracy and stability of volume are not high. Hengwei $\mathrm{Xu}^{[2]}$ has proposed a idea, based on 3D laser scanner to obtain the tree point cloud data as the data source, then use planar scattered point set convex hull algorithm ${ }^{[3]}$ and irregular slices segmentation accumulation algorithm to extract canopy projection area and canopy volume automatically .however this method uses planar convex hull algorithm, the section area is slightly larger than the actual area, increase the computational error. Jinling Niu ${ }^{[4]}$ proposed using shape from focus ${ }^{[5]}$ algorithm to obtain 3D point data and using marching cubes [6] algorithm for hexahedral partition and modeling of objects, accumulate small cube size to obtain results, this method ${ }^{[7]}$ needs to modeling and division, increases the computational complexity, and it is too complicated for the non-scattered point cloud data On the basis of this paper, some scholars have proposed to use marching cube algorithm for reduction and calculation of data, and use contour information to calculate the volume, this method has the contour search process, increasing the difficulty of calculation and time.

There exists insufficient based on the above method currently, for the precision and efficiency of point cloud volume calculation, this paper puts forward to filter the projecting plane data point using scanning algorithm ,restore the outline of projective planes effectively and accurately using edge feature points, then seek the point cloud volume.

\section{Volume Calculation Algorithm}

In this paper, data from the point cloud data acquisition system, which consists of scanning subsystem, rotation subsystem and control subsystem. The measured object is placed on a rotating platform of rotation system, by controlling laser scanning subsystem scan the measured object to obtain the point cloud data. The flow chart is shown in Fig.1.

Point cloud data which is obtained by the laser point cloud data acquisition system describes 3D contour of the measured object, this method chooses arbitrary the rotation platform as the projection plane, and the rotation axis as the $Z$-axis projection coordinates, then chooses two vertical vectors on the projection plane as the $X$-axis and $Y$-axis. The point cloud data to be cut in the $Z$-axis direction, When the data is in a given data area( $\min \langle Z<\min +d * s 1)$ After data is stored, project these data on the projection plane ,then screen the data which is projected, finally calculate it's area. Repeat the above steps until finish to screen all data. 


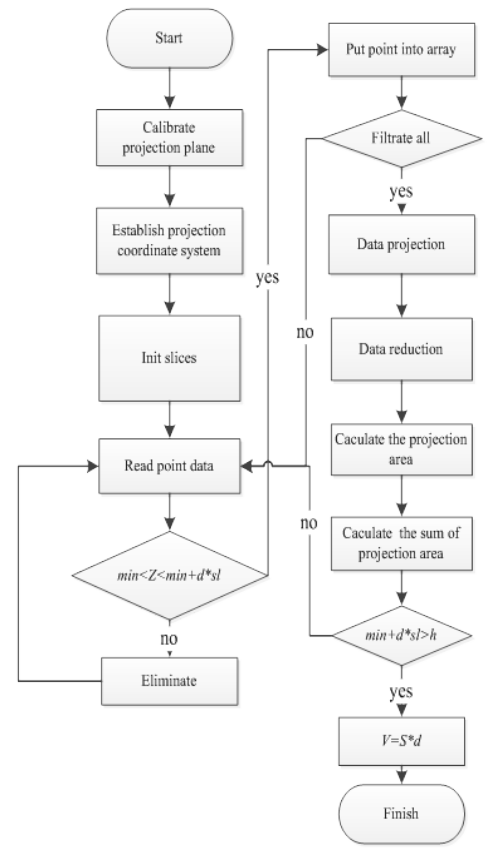

Fig. 1. volume calculation process

\section{A. Projection Plane}

Measured object placed on the rotating platform to scan , this paper put the rotation plane as a projection plane during the rotating axis calibration process, it is assumed binocular camera has been calibrated, Mark the point on the plane, and rotate twice for some angle, then respectively mark position after rotation to obtain three points, denote by $Q_{1}\left(x_{1}, y_{1}, z_{1}\right)$, $Q_{2}\left(x_{2}, y_{2}, z_{2}\right), Q_{3}\left(x_{3}, y_{3}, z_{3}\right)$, principle is shown in Fig.2.

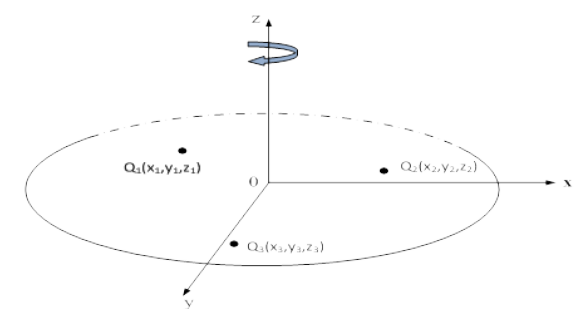

Fig. 2. Determined schematic projection plane

Obviously, these the plane which is determined by these three points three points is the plane of rotation, namely the projection plane:

$$
\begin{aligned}
& {\left[\left(y_{2}-y_{1}\right)\left(z_{3}-z_{1}\right)-\left(y_{3}-y_{1}\right)\left(z_{2}-z_{1}\right)\right]\left(x-x_{1}\right)} \\
& +\left[\left(x_{3}-x_{1}\right)\left(z_{2}-z_{1}\right)-\left(x_{2}-x_{1}\right)\left(z_{3}-z_{1}\right)\right]\left(y-y_{1}\right) \\
& +\left[\left(x_{2}-x_{1}\right)\left(y_{3}-y_{1}\right)-\left(y_{3}-y_{1}\right)\left(z_{2}-z_{1}\right)\right]\left(z-z_{1}\right)=0
\end{aligned}
$$

\section{B. Projection Plane Coordinate System}

When the point cloud is cut, it needs to be cut in one direction, so it is necessary to establish a projection plane coordinate system, which is called projection coordinate system. The normal vector of the projection plane is used as the $Z$-axis, and put any vector on a projection plane perpendicular to the normal vector.

$$
\mathrm{Q}_{1}\left(\mathrm{x}_{1}, \mathrm{y}_{1}, \mathrm{z}_{1}\right), \mathrm{Q}_{2}\left(\mathrm{x}_{2}, \mathrm{y}_{2}, \mathrm{z}_{2}\right), \mathrm{Q}_{3}\left(\mathrm{x}_{3}, \mathrm{y}_{3}, \mathrm{z}_{3}\right) \text { constitute }
$$

two vectors $Q_{1} Q_{2}, Q_{1} Q_{3}$, then get the normal vector:

$$
\mathrm{E}_{\mathrm{Z}}=\mathrm{Q}_{1} \mathrm{Q}_{2} \times \mathrm{Q}_{1} \mathrm{Q}_{3}
$$

That is:

$$
\begin{aligned}
& E_{z}=\left[\left(\left(y_{2}-y_{1}\right)\left(z_{3}-z_{1}\right)-\left(y_{3}-y_{1}\right)\left(z_{2}-z_{1}\right)\right),\right. \\
& \left(\left(x_{3}-x_{1}\right)\left(z_{2}-z_{1}\right)-\left(x_{2}-x_{1}\right)\left(z_{3}-z_{1}\right)\right), \\
& \left.\left(\left(x_{2}-x_{1}\right)\left(y_{3}-y_{1}\right)-\left(y_{3}-y_{1}\right)\left(z_{2}-z_{1}\right)\right)\right]
\end{aligned}
$$

According to that three points can determine rotates to form a circle, denoted by $0(\mathrm{a}, \mathrm{b}, \mathrm{c})$, using the properties of a circle, Any two points on a circle perpendicular bisector connection over the center, still take $\mathrm{Q}_{1} \mathrm{Q}_{2}, \mathrm{Q}_{1} \mathrm{Q}_{3}$, The midpoint of the perpendicular bisectors is denoted $m_{1}, m_{2}$ respectively, In addition, it can also draw the connection between point and the center of the circle, through three vertical relationship:

$$
\begin{gathered}
\mathrm{Q}_{1} \mathrm{Q}_{2} \cdot \mathrm{m}_{1} \mathrm{O}=0 \\
\mathrm{Q}_{2} \mathrm{Q}_{3} \cdot \mathrm{m}_{2} \mathrm{O}=0 \\
\mathrm{Q}_{1} \mathrm{O} \cdot \mathrm{R}=0
\end{gathered}
$$

From (4),(5),(6),we have:

$$
\left[\begin{array}{ccc}
x_{2}-x_{1} & y_{2}-y_{1} & z_{2}-z_{1} \\
x_{3}-x_{2} & y_{3}-y_{2} & z_{3}-z_{2} \\
a & b & c
\end{array}\right]\left[\begin{array}{l}
a \\
b \\
c
\end{array}\right]=\left[\begin{array}{c}
\frac{1}{2}\left[x_{2}^{2}+y_{2}^{2}+z_{2}^{2}-\left(x_{1}^{2}+y_{1}^{2}+z_{1}^{2}\right)\right] \\
\frac{1}{2}\left[x_{3}^{2}+y_{3}^{2}+z_{3}^{2}-\left(x_{2}^{2}+y_{2}^{2}+z_{2}^{2}\right)\right] \\
a x_{1}+b y_{1}+c z_{1}
\end{array}\right]
$$

The $X$-axis unit vector:

$$
\mathrm{E}_{\mathrm{X}}=\frac{\mathrm{Q}_{1} \mathrm{O}}{\left|\mathrm{Q}_{1} \mathrm{O}\right|}
$$

$Y$-axis unit vector:

$$
\mathrm{E}_{\mathrm{Y}}=\mathrm{E}_{\mathrm{Z}} \times \mathrm{E}_{\mathrm{X}}
$$

Determine the projected coordinate system.by formula (2), (8), (9).

\section{C. point cloud projection}

In general, the projection means a reduced dimensional operation, After data has dimensional reduction, it can greatly reduce the complexity of the calculations. It can be used to zoom in or out of the object by scaling factor $K$, if it has to "squeeze" or "stretch" the object, just apply different factors in different directions, if $|k|<1$, objects will be "shorter"; if $|k|>1$, object will be "longer"; if $|k|=0$, called the object projected on a coordinate plane or axis is orthogonal projection. By making the scaling factor in the vertical direction to zero, it can be projected onto the plane:

$$
\mathrm{P}_{\mathrm{xy}}=\mathrm{S}([0,0,1], 0)=\left[\begin{array}{lll}
1 & 0 & 0 \\
0 & 1 & 0 \\
0 & 0 & 0
\end{array}\right]
$$


where $P_{x y}$ is projection matrix, $S([0,0,1], 0)$ is a scaling factor on the direction of rotation $k=0$.Let's denote 3D coordinate by $p_{t r}=\left(\mathrm{x}_{\mathrm{tr}}, \mathrm{y}_{\mathrm{tr}}, \mathrm{z}_{\mathrm{tr}}\right)$, denote 2D coordinate by $p_{t w}$, so:

$$
\mathrm{p}_{\mathrm{tw}}=\mathrm{p}_{\mathrm{tr}} \times \mathrm{P}_{\mathrm{xy}}
$$

The measured object's point cloud data can be cut along the $Z$-axis direction to be projected in the $X O Y$ plane respectively at the same time, and the size of the cut impact on the final volume of precision.

\section{D. projection area calculation}

The core algorithm of this method is to calculate the area of the projection area. After having finished the construction of the projection plane, the area of the projection plane is calculated, and the whole calculation process is shown in Fig 3.

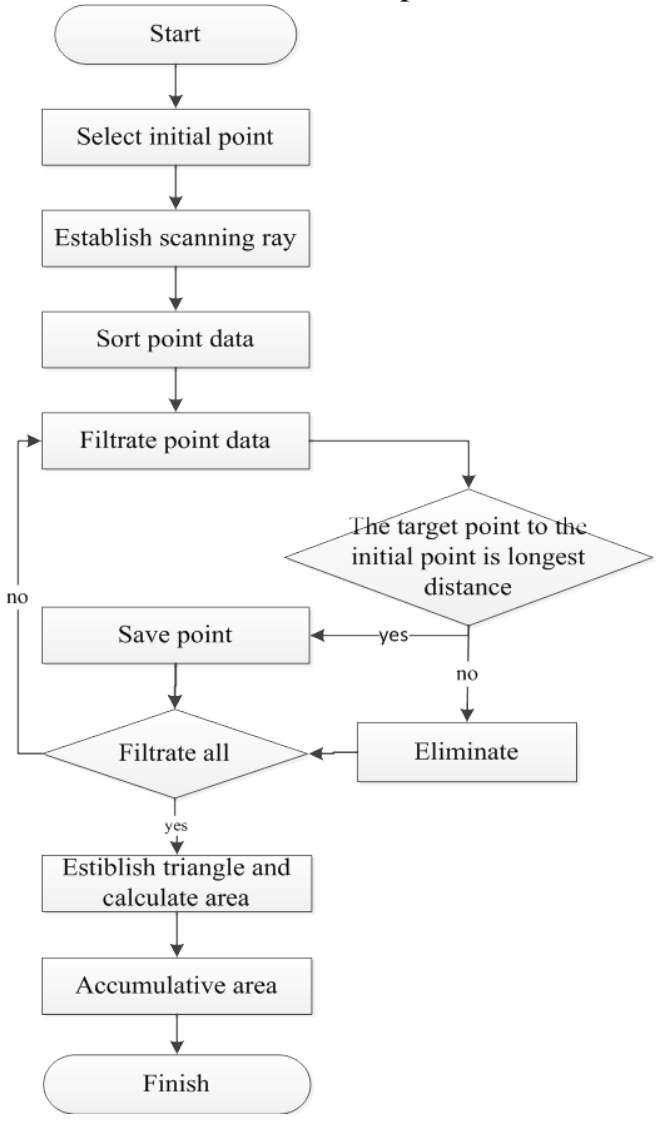

Fig. 3. projected area calculation flowchart

As shown in Fig 3, the algorithm is mainly divided into the following steps:

1) Select the initial point.Firstly ,search the minimum and maximum values of the cloud horizontal direction and the minimum and maximum value of the vertical direction in all of the 2D projection point data.then, construct a square containing all points;finall, the initial point is the intersection of the two diagonal lines of the square, and find the bottom left point, denoted by $A$;

2) Construct a ray with the initial point,and make the and counter clockwise rotation with the initial scan line which is the ray when the angle with the $X$-axis at 0 ;

3) Sort the angles of the lines ,between all cloud data points and the initial point, and $X$-axis from small to big according to the angle;

4) Scan with the scan line, when the same angle rays contain two or more points, select the outermost point (judging by distances), leaving only the outermost points.

5) Construct triangles in turn in counterclockwise direction with the connection of point $A$ and the remaining points, and get the triangle area with vectors;

6) Calculate the area of the projection surface by accumulating the area of all triangles.

7) Followed by all the projection area, multiplied by the height of the slice, and concluded that point size.

In the fourth step, the algorithm process select the elimination to the 2D point cloud data , from the initial light Ray scans the entire point cloud, points out the data points near the beginning, when the area is calculated , the amount of data is greatly reduced.

\section{EXPERIMENT RESULTS AND ERROR ANALYSIS}

To verify the accuracy and efficiency of the point cloud volume calculation algorithms, a few experiments were designed to test the method of this paper, and compare the results with two methods usually used nowadays. in generally, people use MATLAB to solve point cloud volume and projection method .As for the spatial point cloud volume algorithm in MATLAB, first of all, use vpolyhedron polyhedral function to form vpolyhedron, Delaunay triangulation the surface of the polyhedron by DelaunayTri function , then get the volume. Projection also use logarithmic stronghold projection by slice method to project the data points, and build two dimensional convex hull when calculating the area of the plane, and plane area is equal to the convex hull area. This method represents the point cloud volume typical algorithm.in literature [3]. As shown in table 1, we used three different methods to compare the regular contour (hot water bottle packaging, glass packaging) with irregular contour (Winnie the pooh artwork) volume. Compared with MATLAB and projection (based on twodimensional convex hull) this method greatly improves the accuracy of measurement, and it is concluded that the method has high stability and universality. By comparing these three measured object. 
TABLE I. VOLUME MEASUREMENT DATA AND ACCURACY COMPARISON

\begin{tabular}{|c|c|c|c|c|c|c|c|}
\hline $\begin{array}{l}\text { name of } \\
\text { measured } \\
\text { object }\end{array}$ & $\begin{array}{l}\text { manual } \\
\text { measurement } \\
\left(\mathbf{m m}^{3}\right)\end{array}$ & $\begin{array}{l}\text { MATL } \\
\text { AB }\end{array}$ & $\begin{array}{l}\text { percentage } \\
\text { error }(\%)\end{array}$ & $\begin{array}{l}\text { Projection method } \\
\text { (based on 2d } \\
\text { convex } \\
\text { hull) }\left(\mathbf{m m}^{3}\right)\end{array}$ & $\begin{array}{l}\text { Percentage } \\
\text { error }(\%)\end{array}$ & $\begin{array}{l}\text { Proposed } \\
\text { algorithm/ } \\
\left(\mathbf{m m}^{3}\right)\end{array}$ & $\begin{array}{l}\text { Percentage } \\
\text { error (\%) }\end{array}$ \\
\hline $\begin{array}{l}\text { hot water } \\
\text { pot in } \\
\text { packaging }\end{array}$ & 9844112.113 & $\begin{array}{l}1012677 \\
3.597\end{array}$ & 2.88 & 10176133.263 & 3.37 & $\begin{array}{l}9903734.1 \\
71\end{array}$ & 0.61 \\
\hline $\begin{array}{l}\text { glass } \\
\text { packaging }\end{array}$ & 1672500.211 & $\begin{array}{l}1721169 \\
.967\end{array}$ & 2.91 & 1729030.718 & 3.38 & $\begin{array}{l}1683036.9 \\
62\end{array}$ & 0.63 \\
\hline $\begin{array}{l}\text { Winnie the } \\
\text { pooh art }\end{array}$ & 3375962.265 & $\begin{array}{l}3473189 \\
.978\end{array}$ & 2.88 & 3490069.789 & 3.38 & $\begin{array}{l}3397568.4 \\
23\end{array}$ & 0.64 \\
\hline
\end{tabular}

Through the analysis in table 1, the main error source of this method is that the point cloud data collection, there will be a "dirty" data, it had a few influence on the calculation accuracy. Moreover, the size of the section will also have effect on the accuracy of the algorithm results

As shown in Fig 4, hot water bottle packaging using 175500 data points, the glass packaging using 87750 data points, winnie the pooh artwork using 114300 data points, when the data points of measured object decreases, the program run time also declined significantly. With the measured object's accuracy which the measurement system requires changing, run time will occur the linear change, when accuracy is slightly lower, run time will also reduce substantially, As for large data amount of point cloud data, the processing time does not increase exponentially. This method has good flexibility which can completely satisfy the requirements of the system under different precision program. run time also declined significantly.

\section{CONCLUSION}

This paper propose a new algorithm based on slice method, which improves and innovates the calculation of 3D point cloud. As for calculation accuracy, it is unnecessary for the complex calculation process which improves accuracy of the current method and is applies the rules and irregular contour of object .Each system has its own product accuracy requirements, this method can achieve the accuracy requirements of most of the system by adjusting the cut size. This method has been applied in the "commodity over packaging intelligent detection system" with independent research and it returns well. So, this method will have a certain significance to other systems and application and it is used very widely.

\section{ACKNOWLEDGMENT}

The authors would like to thank the anonymous reviewers and the appendices providers. This work is supported by the science and technology plan projects of Xi'an (CXY1441(7)),and the Shaanxi science and technology research and development projects - industrial research (2014 k05-33).

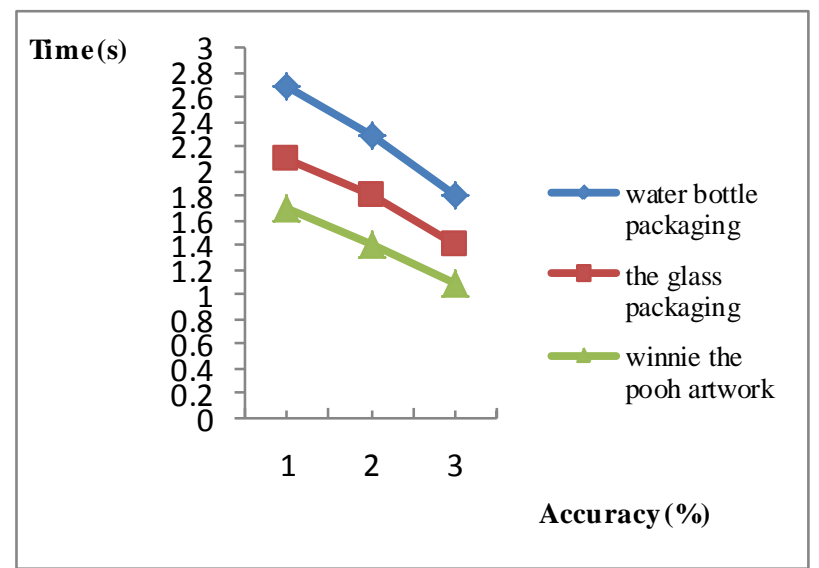

Fig. 4. Accuracy and time line chart

\section{REFERENCES}

[1] Rahman, M.H., Qiang Li , Pickering, M. "Food volume estimation in a mobile phone based dietary assessment system”. Signal Image Technology and Internet Based Systems (SITIS), 2012 Eighth International Conference on. November 2012,pp:988-995.

[2] XU Heng-wei,FENG Zhong-ke,SU Zhi-fang. "An automatic extraction algorithm based on 3D laser point cloud data of single tree canopy projection area and canopy volume". Spectroscopy and Spectral Analysis,vol. 34, pp.465-470, February 2014

[3] Klette, G. "A recursive algorithm for calculating the relative convex hull” Image and Vision Computing New Zealand (IVCNZ), 2010 25th International Conference of.pp.1-7,Noveber.2010

[4] Niu Jinling,Zhang Linpeng. "Study on the method of volume measurement of irregular volume based on scattered data points" The 14th national conference on image graphics,pp.42-46,May 2008

[5] Muhammad Tariq Mahmood, Young-Kyu Choi,” Estimating shape from focus by Gaussian process regression” IEEE International Conference on Systems, Man, and Cybernetics,pp.1345-1350,Octber 2012

[6] Carlos A. Dietrich, Carlos E. Scheidegger, John Schreiner, "Edge transformations for improving mesh quality of marching cubes” pp.150159,February 2009

[7] Shiping Zhu, Jie Gao.”3D modeling and rendering based on uncalibrated single view image in undergraduate final design”, Computer Science and Information Processing (CSIP),International Conference on.pp.13361340,Augest 2012 